\title{
SYYSTEMIC SCLEROSIS
}

Systemic sclerosis is an autoimmune disease characterized by microvascular damage and progressive fibrosis of the skin and visceral organs that might result in life-threatening complications, including renal crisis.

\section{MECHANISMS}

The exact cause of systemic sclerosis remains enigmatic but involves an interplay between genetic factors and environmental events (such as viral infections). Initially, pathogenesis is dominated by microvascular injury, which leads to endothelial cell activation and platelet aggregation. The resulting hypoxia and chemokine release trigger an uncontrolled inflammatory reaction, infiltration of immune cells and secretion of autoantibodies. As a consequence, resident fibroblasts undergo differentiation into myofibroblasts, which are responsible for the excessive extracellular matrix production that underlies the fibrosis.

Several signalling pathways are implicated in this transition, of which transforming growth factor- $\beta$ and activation of Toll-like receptor 4

predominate.

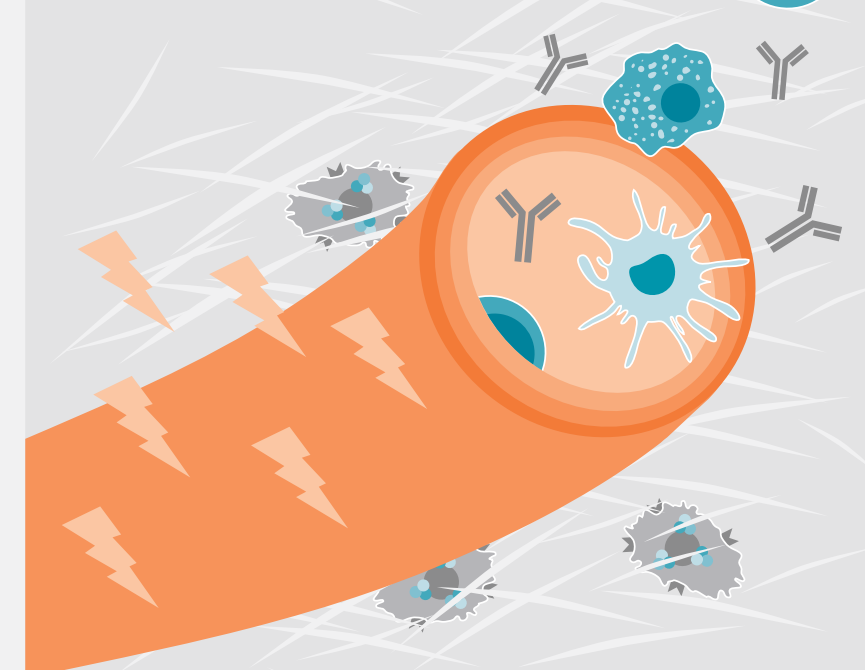

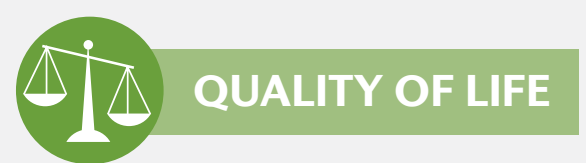

Scarring of

the skin and organs leads to interstitial lung disease, pulmonary arterial hypertension, renal crisis, gastrointestinal complications. Patients' hands often show

Raynaud phenomenon and ulcers
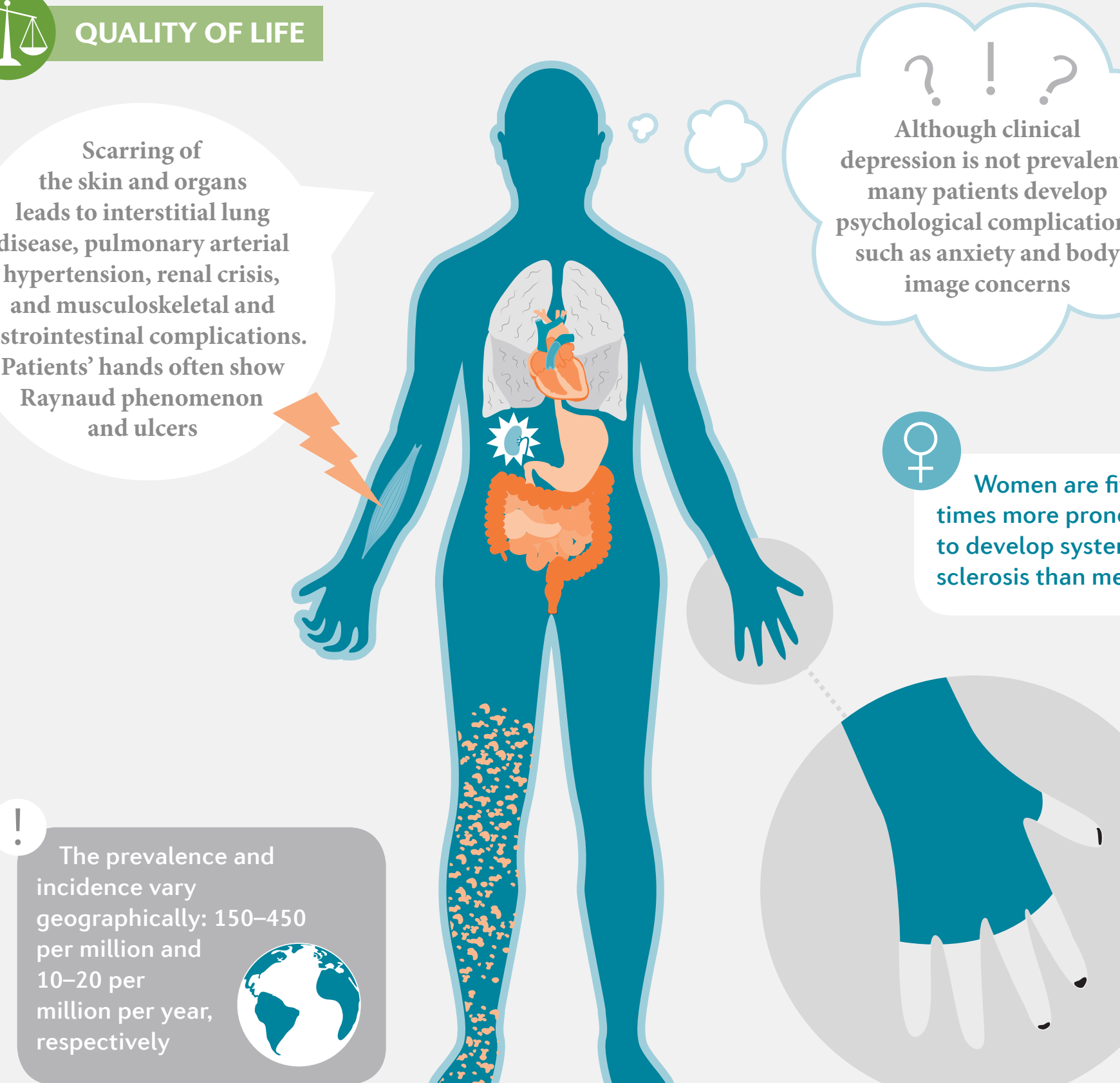
Although clinical depression is not prevalent, many patients develop psychological complications such as anxiety and body image concerns<smiles>C1CCCCC1</smiles>
and musculoskeletal and

For the Primer, visit doi:10.1038/nrdp.2015.2

\section{DIAGNOSIS}

In general, patients are classified on the basis of the location of skin fibrosis, as illustrated below. However, the high interpatient variability makes diagnosis particularly challenging. In 2013, new criteria that take into account accompanying complicatio into account accompanying complications and biomarkers were proposed, although these still lack sensitivity to classify the disease early. Early detection and accurate disease subset definitions are urgently needed to identify patients at risk for specific complications or rapid progression.

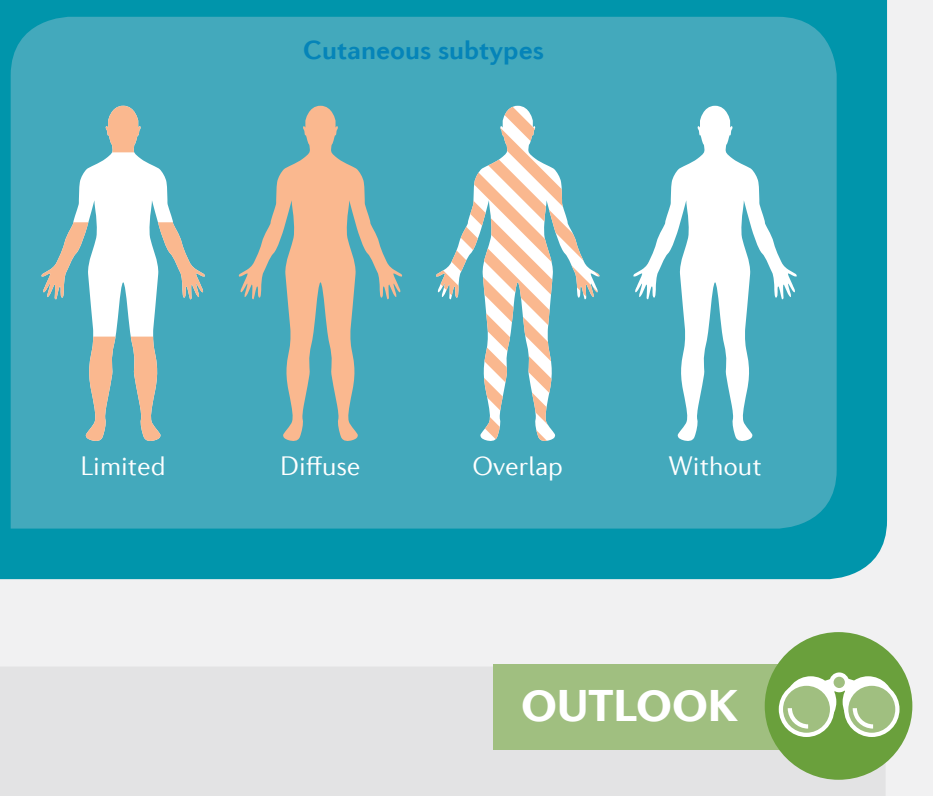

A better understanding of the triggers, cell types and pathways involved in systemic sclerosis will clear the way for the design of new, targeted medication and the repurposing of existing

therapies. In addition, not only is the identification of specific and reliable biomarkers essential to improve early diagnosis, but it will also help to optimize and standardize clinical trial design. The latter is urgently needed to validate potential therapies but is difficult to implement because of confounding factors - including the natural amelioration of symptoms over time and the lack of robust subgroup definitions. as immune suppression and haematopoietic stem cell complication-specific therapies. and fibrotic anomalies. Treatments include immunomodulation (such transplantation), as well as are used to treat hypertension, angiotensin-converting enzyme inhibitors to prevent renal crisis, and endothelin receptor antagonists to treat digital ulcers. 